DOI 10.4171/JEMS/251

Simon Brendle

\title{
A note on Ricci flow and optimal transportation
}

Received September 11, 2009 and in revised form July 10, 2010

Abstract. We describe a new link between Perelman's monotonicity formula for the reduced volume and ideas from optimal transport theory.

\section{Introduction}

In this note, we describe an interpolation inequality in the setting of Ricci flow and $\mathcal{L}$ distance. This inequality is motivated by the following classical inequality due to Prékopa and Leindler:

Theorem 1 (Prékopa [9]; Leindler [5]). Fix a real number $0<\lambda<1$. Moreover, let $u_{1}, u_{2}, v: \mathbb{R}^{n} \rightarrow \mathbb{R}$ be nonnegative measurable functions satisfying

$$
v((1-\lambda) x+\lambda y) \geq u_{1}(x)^{1-\lambda} u_{2}(y)^{\lambda}
$$

for all points $x, y \in \mathbb{R}^{n}$. Then

$$
\int_{\mathbb{R}^{n}} v \geq\left(\int_{\mathbb{R}^{n}} u_{1}\right)^{1-\lambda}\left(\int_{\mathbb{R}^{n}} u_{2}\right)^{\lambda} .
$$

Cordero-Erausquin, McCann, and Schmuckenschläger [2], [3] have generalized this inequality to Riemannian manifolds. The proof employs techniques from optimal transport theory.

Our goal in this paper is to replace the Riemannian distance by Perelman's $\mathcal{L}$-distance (cf. [8]). The theory of $\mathcal{L}$-optimal transport was developed in recent work of Topping [11] (see also [6], [7]). Among other things, Topping proved an important monotonicity formula for the $\mathcal{L}$-Wasserstein distance on the space of probability measures. Lott [6] established a convexity property for the entropy along $\mathcal{L}$-Wasserstein geodesics.

To fix notation, let $M$ be a compact manifold of dimension $n$, and let $g(t), t \in[0, T]$, be a one-parameter family of metrics on $M$. We assume that the metrics $g(t)$ evolve by backward Ricci flow, i.e.

$$
\frac{\partial}{\partial t} g(t)=2 \operatorname{Ric}_{g(t)}
$$

S. Brendle: Department of Mathematics, Stanford University, Stanford, CA 94305, USA; e-mail: brendle@math.stanford.edu 
This evolution equation was introduced in a seminal paper by R. Hamilton [4]. For an introduction to Ricci flow, see e.g. [1] or [10]. Following Perelman [8], we define the $\mathcal{L}$-length of a path $\gamma:\left[\tau_{1}, \tau_{2}\right] \rightarrow M$ by

$$
\mathcal{L}(\gamma)=\int_{\tau_{1}}^{\tau_{2}} \sqrt{t}\left(R_{g(t)}(\gamma(t))+\left|\gamma^{\prime}(t)\right|_{g(t)}^{2}\right) d t
$$

where $R_{g(t)}$ denotes the scalar curvature of the metric $g(t)$. Moreover, the $\mathcal{L}$-distance is defined by

$$
Q\left(x, \tau_{1} ; y, \tau_{2}\right)=\inf \left\{\mathcal{L}(\gamma): \gamma:\left[\tau_{1}, \tau_{2}\right] \rightarrow M, \gamma\left(\tau_{1}\right)=x, \gamma\left(\tau_{2}\right)=y\right\}
$$

A path $\gamma:\left[\tau_{1}, \tau_{2}\right] \rightarrow M$ is called an $\mathcal{L}$-geodesic if the first variation of $\mathcal{L}$ is zero. For each tangent vector $Z \in T_{x} M$, we define

$$
\mathcal{L}_{\tau_{1}, \tau_{2}} \exp _{x}(Z)=\gamma\left(\tau_{2}\right)
$$

where $\gamma:\left[\tau_{1}, \tau_{2}\right] \rightarrow M$ is the unique $\mathcal{L}$-geodesic satisfying $\gamma\left(\tau_{1}\right)=x$ and $\sqrt{\tau_{1}} \gamma^{\prime}\left(\tau_{1}\right)=Z$. The map $\mathcal{L}_{\tau_{1}, \tau_{2}} \exp _{x}: T_{x} M \rightarrow M$ is called the $\mathcal{L}$-exponential map.

The following is the main result of this note:

Theorem 2. Fix real numbers $\tau_{1}, \tau_{2}$, $\tau$ such that $0<\tau_{1}<\tau<\tau_{2}<T$. For abbreviation, we write

$$
\frac{1}{\sqrt{\tau}}=\frac{1-\lambda}{\sqrt{\tau_{1}}}+\frac{\lambda}{\sqrt{\tau_{2}}}
$$

where $0<\lambda<1$. Let $u_{1}, u_{2}, v: M \rightarrow \mathbb{R}$ be nonnegative measurable functions such that

$$
\begin{aligned}
\left(\frac{\tau}{\tau_{1}^{1-\lambda} \tau_{2}^{\lambda}}\right)^{n / 2} v(\gamma(\tau)) \geq & \exp \left(-\frac{1-\lambda}{2 \sqrt{\tau_{1}}} Q\left(\gamma\left(\tau_{1}\right), \tau_{1} ; \gamma(\tau), \tau\right)\right) u_{1}\left(\gamma\left(\tau_{1}\right)\right)^{1-\lambda} \\
& \cdot \exp \left(\frac{\lambda}{2 \sqrt{\tau_{2}}} Q\left(\gamma(\tau), \tau ; \gamma\left(\tau_{2}\right), \tau_{2}\right)\right) u_{2}\left(\gamma\left(\tau_{2}\right)\right)^{\lambda}
\end{aligned}
$$

for every minimizing $\mathcal{L}$-geodesic $\gamma:\left[\tau_{1}, \tau_{2}\right] \rightarrow M$. Then

$$
\int_{M} v d \operatorname{vol}_{g(\tau)} \geq\left(\int_{M} u_{1} d \operatorname{vol}_{g\left(\tau_{1}\right)}\right)^{1-\lambda}\left(\int_{M} u_{2} d \operatorname{vol}_{g\left(\tau_{2}\right)}\right)^{\lambda}
$$

By sending $\tau_{1} \rightarrow 0$, we recover the monotonicity of Perelman's reduced volume. This is discussed in Section 3 


\section{Proof of Theorem 2}

In order to prove Theorem 2, we make extensive use of Topping's notion of $\mathcal{L}$-optimal transportation (cf. [11]; see also [6]). Without loss of generality, we may assume that

$$
\int_{M} u_{1}(x) d \operatorname{vol}_{g\left(\tau_{1}\right)}=\int_{M} u_{2}(x) d \operatorname{vol}_{g\left(\tau_{2}\right)}=1 .
$$

We define Borel probability measures $v_{1}$ and $v_{2}$ by

$$
d \nu_{1}=u_{1} d \operatorname{vol}_{g\left(\tau_{1}\right)} \quad \text { and } \quad d \nu_{2}=u_{2} d \operatorname{vol}_{g\left(\tau_{2}\right)} .
$$

Topping introduced a notion of reflexive function (see [11, Definition 2.1] for a precise definition). This notion is analogous to the concept of $c$-concavity in the classical theory of optimal transportation. Topping then proved the following existence theorem (cf. [11, Section 2]; see also [12, Theorem 10.28]):

Proposition 3 (P. Topping [11]). We can find a reflexive function $\varphi: M \rightarrow \mathbb{R}$, a Borel set $K \subset M$, and a Borel map $F: M \rightarrow M$ with the following properties:

(i) $\nu_{2}=F_{\#} \nu_{1}$.

(ii) The set $M \backslash K$ has measure zero.

(iii) The function $\varphi$ is differentiable at each point $x \in K$.

(iv) If $x \in K$ and $y=F(x)$, then the function $Q\left(\cdot, \tau_{1} ; y, \tau_{2}\right)-\varphi$ attains its global minimum at the point $x$. In particular, the function $Q\left(\cdot, \tau_{1} ; y, \tau_{2}\right)-\varphi$ is differentiable at $x$, and its gradient is equal to zero.

For each $t \in\left[\tau_{1}, \tau_{2}\right]$, we define a Borel map $F_{t}: M \rightarrow M$ by

$$
F_{t}(x)=\mathcal{L}_{\tau_{1}, t} \exp _{x}\left(-\frac{1}{2} \nabla \varphi(x)\right)
$$

for $x \in K$. The following result is a consequence of property (iv) in Proposition 3 (cf. [11, Lemma 2.4]).

Proposition 4. We have $F_{\tau_{1}}(x)=x$ and $F_{\tau_{2}}(x)=F(x)$ for all $x \in K$. Moreover, for each point $x \in K$, the path $t \mapsto F_{t}(x)$ has minimal $\mathcal{L}$-length among all paths joining $\left(x, \tau_{1}\right)$ and $\left(F(x), \tau_{2}\right)$.

Since $\varphi$ is reflexive, the function $\varphi$ is semiconcave (cf. [11, Lemma 2.10]). By Theorem 14.1 in [12], we can find a Borel set $\tilde{K} \subset K$ with the following properties:

- The set $M \backslash \tilde{K}$ has measure zero.

- For each point $x \in \tilde{K}$, the function $\varphi$ admits a Taylor expansion of order two around $x$. 
For each point $x \in \tilde{K}$, we denote by $\nabla \varphi(x)$ and (Hess $\varphi)_{x}$ the gradient and Hessian of the function $\varphi$ with respect to the metric $g\left(\tau_{1}\right)$. Theorem 14.1 in [12] guarantees that $(\operatorname{Hess} \varphi)_{x}$ is symmetric.

We next describe the volume distortion coefficients associated with the map $F_{t}$. To that end, we fix a point $x \in \tilde{K}$ and a time $t \in\left(\tau_{1}, \tau_{2}\right]$. The linearization of the $\mathcal{L}$ exponential map $\mathcal{L}_{\tau_{1}, t} \exp _{x}$ gives a linear transformation

$$
D\left(\mathcal{L}_{\tau_{1}, t} \exp _{x}\right)_{-\frac{1}{2} \nabla \varphi(x)}:\left(T_{x} M, g\left(\tau_{1}\right)\right) \rightarrow\left(T_{F_{t}(x)} M, g(t)\right) .
$$

Moreover, the Hessian of the function $Q\left(\cdot, \tau_{1} ; F_{t}(x), t\right)-\varphi$ at the point $x$ defines a symmetric linear transformation from the tangent space $\left(T_{x} M, g\left(\tau_{1}\right)\right)$ into itself.

Let us denote by $\Psi_{x, t}:\left(T_{x} M, g\left(\tau_{1}\right)\right) \rightarrow\left(T_{F_{t}(x)} M, g(t)\right)$ the composition of these two linear transformations; that is,

$$
\Psi_{x, t}=\frac{1}{2} D\left(\mathcal{L}_{\tau_{1}, t} \exp _{x}\right)_{-\frac{1}{2} \nabla \varphi(x)} \circ\left[\operatorname{Hess}\left(Q\left(\cdot, \tau_{1} ; F_{t}(x), t\right)-\varphi\right)\right]_{x}
$$

(cf. [11, Lemma 2.13]). We note that $\Psi_{x, t}$ can be characterized in terms of $\mathcal{L}$-Jacobi fields; see [11, Lemma 2.18] for details. Finally, we define the volume distortion coefficients by

$$
\mathcal{J}(x, t)=\operatorname{det} \Psi_{x, t}
$$

for each point $x \in \tilde{K}$ and each $t \in\left(\tau_{1}, \tau_{2}\right]$.

Proposition 5. For each point $x \in \tilde{K}$, we have

$$
\begin{aligned}
\tau^{-n / 2} \exp ( & \left.-\frac{1-\lambda}{2 \sqrt{\tau_{1}}} Q\left(x, \tau_{1} ; F_{\tau}(x), \tau\right)\right) \mathcal{J}(x, \tau) \\
& \geq \tau_{1}^{-n(1-\lambda) / 2} \tau_{2}^{-n \lambda / 2} \exp \left(-\frac{\lambda}{2 \sqrt{\tau_{2}}} Q\left(F_{\tau}(x), \tau ; F(x), \tau_{2}\right)\right) \mathcal{J}\left(x, \tau_{2}\right)^{\lambda}
\end{aligned}
$$

Proof. Fix a point $x \in \tilde{K}$, and let $\left\{e_{1}, \ldots, e_{n}\right\}$ be a basis of $T_{x} M$ which is orthonormal with respect to the metric $g\left(\tau_{1}\right)$. We next consider the path

$$
\gamma:\left[\tau_{1}, \tau_{2}\right] \rightarrow M, \quad t \mapsto F_{t}(x) .
$$

By Lemma 4, the path $\gamma$ has minimal $\mathcal{L}$-length among all paths joining $\left(x, \tau_{1}\right)$ to $\left(F(x), \tau_{2}\right)$.

Let $\left\{E_{1}(t), \ldots, E_{n}(t)\right\}$ be vector fields along $\gamma$ such that $E_{i}\left(\tau_{1}\right)=e_{i}$ and

$$
\left\langle D_{t} E_{i}(t), E_{j}(t)\right\rangle_{g(t)}+\operatorname{Ric}_{g(t)}\left(E_{i}(t), E_{j}(t)\right)=0
$$

for all $t \in\left[\tau_{1}, \tau_{2}\right]$. For each $t \in\left[\tau_{1}, \tau_{2}\right]$, the vectors $\left\{E_{1}(t), \ldots, E_{n}(t)\right\}$ are orthonormal with respect to the metric $g(t)$.

Let $\left\{Y_{1}(t), \ldots, Y_{n}(t)\right\}$ be $\mathcal{L}$-Jacobi fields along $\gamma$ satisfying the initial conditions

$$
Y_{j}\left(\tau_{1}\right)=e_{j} \quad \text { and } \quad\left\langle e_{i}, D_{t} Y_{j}\left(\tau_{1}\right)\right\rangle_{g\left(\tau_{1}\right)}=-\frac{1}{2 \sqrt{\tau_{1}}}(\operatorname{Hess} \varphi)_{x}\left(e_{i}, e_{j}\right)
$$


For each $t \in\left[\tau_{1}, \tau_{2}\right]$, we define an $n \times n$ matrix $A(t)$ by

$$
a_{i j}(t)=\left\langle E_{i}(t), Y_{j}(t)\right\rangle_{g(t)}
$$

It follows from the initial conditions for $Y_{j}$ that $a_{i j}\left(\tau_{1}\right)=\delta_{i j}$ and

$$
a_{i j}^{\prime}\left(\tau_{1}\right)=\operatorname{Ric}_{g\left(\tau_{1}\right)}\left(e_{i}, e_{j}\right)-\frac{1}{2 \sqrt{\tau_{1}}}(\operatorname{Hess} \varphi)_{x}\left(e_{i}, e_{j}\right) .
$$

In particular, the matrix $A^{\prime}\left(\tau_{1}\right) A\left(\tau_{1}\right)^{-1}$ is symmetric. Moreover, it was shown by Topping [11] that

$$
A^{\prime \prime}(t)+\frac{1}{2 t} A^{\prime}(t)=M(t) A(t)
$$

for all $t \in\left[\tau_{1}, \tau_{2}\right]$. Here, $M(t)$ is a symmetric $n \times n$ matrix, whose trace is given by

$$
\begin{aligned}
2 \operatorname{tr}(M(t))= & \frac{\partial}{\partial t} R_{g(t)}(\gamma(t))+2\left\langle\nabla R_{g(t)}(\gamma(t)), \gamma^{\prime}(t)\right\rangle_{g(t)} \\
& -2 \operatorname{Ric}_{g(t)}\left(\gamma^{\prime}(t), \gamma^{\prime}(t)\right)+\frac{1}{t} R_{g(t)}(\gamma(t)) .
\end{aligned}
$$

Arguing as in the proof of Lemma 3.1 in [11], we obtain

$$
\begin{aligned}
t^{-3 / 2} \frac{d}{d t}[ & \left.t^{3 / 2} \frac{d}{d t} \log \operatorname{det} A(t)\right] \\
& =\frac{d^{2}}{d t^{2}} \log \operatorname{det} A(t)+\frac{3}{2 t} \frac{d}{d t} \log \operatorname{det} A(t) \\
& =\operatorname{tr}\left(A^{\prime \prime}(t) A(t)^{-1}\right)-\operatorname{tr}\left(A^{\prime}(t) A(t)^{-1} A^{\prime}(t) A(t)^{-1}\right)+\frac{3}{2 t} \operatorname{tr}\left(A^{\prime}(t) A(t)^{-1}\right) \\
& =\operatorname{tr}(M(t))-\operatorname{tr}\left(A^{\prime}(t) A(t)^{-1} A^{\prime}(t) A(t)^{-1}\right)+\frac{1}{t} \operatorname{tr}\left(A^{\prime}(t) A(t)^{-1}\right) \\
& =\operatorname{tr}(M(t))-\operatorname{tr}\left[\left(A^{\prime}(t) A(t)^{-1}-\frac{1}{2 t} I\right)^{2}\right]+\frac{n}{4 t^{2}}
\end{aligned}
$$

Note that the matrix $A^{\prime}\left(\tau_{1}\right) A\left(\tau_{1}\right)^{-1}$ is symmetric. Moreover, the matrix $M(t)$ is symmetric for each $t \in\left[\tau_{1}, \tau_{2}\right]$. Consequently, the matrix $A^{\prime}(t) A(t)^{-1}$ is symmetric for all $t \in\left[\tau_{1}, \tau_{2}\right]$. Hence, we obtain

$$
t^{-3 / 2} \frac{d}{d t}\left[t^{3 / 2} \frac{d}{d t} \log \operatorname{det} A(t)\right] \leq \operatorname{tr}(M(t))+\frac{n}{4 t^{2}}
$$

for all $t \in\left[\tau_{1}, \tau_{2}\right]$. On the other hand, we have

$$
\frac{d}{d t} Q\left(x, \tau_{1} ; F_{t}(x), t\right)=\sqrt{t}\left(R_{g(t)}(\gamma(t))+\left|\gamma^{\prime}(t)\right|_{g(t)}^{2}\right)
$$


by definition of the $\mathcal{L}$-distance. This implies

$$
\begin{aligned}
t^{-3 / 2} & \frac{d}{d t}\left[t^{3 / 2} \frac{d}{d t}\left(t^{-1 / 2} Q\left(x, \tau_{1} ; F_{t}(x), t\right)\right)\right] \\
= & t^{-1} \frac{d}{d t}\left[t^{1 / 2} \frac{d}{d t} Q\left(x, \tau_{1} ; F_{t}(x), t\right)\right]=t^{-1} \frac{d}{d t}\left[t\left(R_{g(t)}(\gamma(t))+\left|\gamma^{\prime}(t)\right|_{g(t)}^{2}\right)\right] \\
& =\frac{\partial}{\partial t} R_{g(t)}(\gamma(t))+2\left\langle\nabla R_{g(t)}(\gamma(t)), \gamma^{\prime}(t)\right\rangle_{g(t)}-2 \operatorname{Ric}_{g(t)}\left(\gamma^{\prime}(t), \gamma^{\prime}(t)\right)+\frac{1}{t} R_{g(t)}(\gamma(t))
\end{aligned}
$$

(cf. [8, equation (7.3)]). Using (1), we obtain

$$
t^{-3 / 2} \frac{d}{d t}\left[t^{3 / 2} \frac{d}{d t}\left(t^{-1 / 2} Q\left(x, \tau_{1} ; F_{t}(x), t\right)\right)\right]=2 \operatorname{tr}(M(t)) .
$$

Putting these facts together, we conclude that

$$
t^{-3 / 2} \frac{d}{d t}\left[t^{3 / 2} \frac{d}{d t}\left(\frac{n}{2} \log t+\frac{1}{2} t^{-1 / 2} Q\left(x, \tau_{1} ; F_{t}(x), t\right)-\log \operatorname{det} A(t)\right)\right] \geq 0 .
$$

Hence, if we write

$$
\frac{n}{2} \log t+\frac{1}{2} t^{-1 / 2} Q\left(x, \tau_{1} ; F_{t}(x), t\right)-\log \operatorname{det} A(t)=h\left(t^{-1 / 2}\right),
$$

then the function $h$ is convex. Using the relation $\tau^{-1 / 2}=(1-\lambda) \tau_{1}^{-1 / 2}+\lambda \tau_{2}^{-1 / 2}$, we obtain

$$
h\left(\tau^{-1 / 2}\right) \leq(1-\lambda) h\left(\tau_{1}^{-1 / 2}\right)+\lambda h\left(\tau_{2}^{-1 / 2}\right),
$$

hence

$$
\begin{aligned}
\tau^{-n / 2} \exp (- & \left.\frac{1}{2 \sqrt{\tau}} Q\left(x, \tau_{1} ; F_{\tau}(x), \tau\right)\right) \operatorname{det} A(\tau) \\
& \geq \tau_{1}^{-n(1-\lambda) / 2} \tau_{2}^{-n \lambda / 2} \exp \left(-\frac{\lambda}{2 \sqrt{\tau_{2}}} Q\left(x, \tau_{1} ; F(x), \tau_{2}\right)\right)\left(\operatorname{det} A\left(\tau_{2}\right)\right)^{\lambda}
\end{aligned}
$$

Moreover, we have

$$
Q\left(x, \tau_{1} ; F(x), \tau_{2}\right)=Q\left(x, \tau_{1} ; F_{\tau}(x), \tau\right)+Q\left(F_{\tau}(x), \tau ; F(x), \tau_{2}\right)
$$

since $\gamma$ is a minimizing $\mathcal{L}$-geodesic. Hence, we obtain

$$
\begin{aligned}
\tau^{-n / 2} \exp & \left(-\frac{1-\lambda}{2 \sqrt{\tau_{1}}} Q\left(x, \tau_{1} ; F_{\tau}(x), \tau\right)\right) \operatorname{det} A(\tau) \\
& \geq \tau_{1}^{-n(1-\lambda) / 2} \tau_{2}^{-n \lambda / 2} \exp \left(-\frac{\lambda}{2 \sqrt{\tau_{2}}} Q\left(F_{\tau}(x), \tau ; F(x), \tau_{2}\right)\right)\left(\operatorname{det} A\left(\tau_{2}\right)\right)^{\lambda}
\end{aligned}
$$

On the other hand, it follows from Lemma 2.18 in [11] that $\Psi_{x, t}\left(e_{j}\right)=Y_{j}(t)$ for all $t \in\left(\tau_{1}, \tau_{2}\right]$. From this, we deduce that $\left\langle E_{i}(t), \Psi_{x, t}\left(e_{j}\right)\right\rangle_{g(t)}=a_{i j}(t)$, hence $\mathcal{J}(x, t)=$ 
$\operatorname{det} A(t)$ for all $t \in\left(\tau_{1}, \tau_{2}\right]$. Putting these facts together, the assertion follows. This completes the proof of Proposition 5 .

We next consider the interpolant measure $v=\left(F_{\tau}\right)_{\#} v_{1}$. It follows from work of Topping that $v$ is absolutely continuous with respect to the volume measure (cf. [11, Lemma 2.17]). Hence, we may write $d v=u d \operatorname{vol}_{g(\tau)}$ for some Borel measurable function $u$. Using Proposition 5, we obtain a lower bound for the density $u$.

Proposition 6. There exists a Borel set $\hat{K} \subset \tilde{K}$ such that $M \backslash \hat{K}$ has measure zero and

$$
\begin{aligned}
\left(\frac{\tau}{\tau_{1}^{1-\lambda} \tau_{2}^{\lambda}}\right)^{n / 2} u\left(F_{\tau}(x)\right) \leq & \exp \left(-\frac{1-\lambda}{2 \sqrt{\tau_{1}}} Q\left(x, \tau_{1} ; F_{\tau}(x), \tau\right)\right) u_{1}(x)^{1-\lambda} \\
& \cdot \exp \left(\frac{\lambda}{2 \sqrt{\tau_{2}}} Q\left(F_{\tau}(x), \tau ; F(x), \tau_{2}\right)\right) u_{2}(F(x))^{\lambda}
\end{aligned}
$$

for all $x \in \hat{K}$.

Proof. It follows from Theorem 2.14 in [11] that

$$
u_{1}(x)=u_{2}(F(x)) \mathcal{J}\left(x, \tau_{2}\right)>0
$$

for almost all $x \in \tilde{K}$. Applying the analogous reasoning to the interpolant measure $v$ yields

$$
u_{1}(x)=u\left(F_{\tau}(x)\right) \mathcal{J}(x, \tau)>0
$$

for almost all $x \in \tilde{K}$. Using Proposition 5 , we obtain

$$
\begin{aligned}
\tau^{-n / 2} \exp \left(-\frac{1-\lambda}{2 \sqrt{\tau_{1}}} Q\left(x, \tau_{1} ; F_{\tau}(x), \tau\right)\right) \frac{u_{1}(x)}{u\left(F_{\tau}(x)\right)} \\
\quad \geq \tau_{1}^{-n(1-\lambda) / 2} \tau_{2}^{-n \lambda / 2} \exp \left(-\frac{\lambda}{2 \sqrt{\tau_{2}}} Q\left(F_{\tau}(x), \tau ; F(x), \tau_{2}\right)\right)\left(\frac{u_{1}(x)}{u_{2}(F(x))}\right)^{\lambda}
\end{aligned}
$$

for almost all $x \in \tilde{K}$. Rearranging terms, the assertion follows.

Corollary 7. We have

$$
\int_{M} v d \operatorname{vol}_{g(\tau)} \geq 1 .
$$

Proof. Fix a point $x \in \hat{K}$. By Lemma 4 , the path $t \mapsto F_{t}(x)$ is a minimizing $\mathcal{L}$-geodesic. Therefore, we have

$$
\begin{aligned}
\left(\frac{\tau}{\tau_{1}^{1-\lambda} \tau_{2}^{\lambda}}\right)^{n / 2} v\left(F_{\tau}(x)\right) \geq & \exp \left(-\frac{1-\lambda}{2 \sqrt{\tau_{1}}} Q\left(x, \tau_{1} ; F_{\tau}(x), \tau\right)\right) u_{1}(x)^{1-\lambda} \\
& \cdot \exp \left(\frac{\lambda}{2 \sqrt{\tau_{2}}} Q\left(F_{\tau}(x), \tau ; F(x), \tau_{2}\right)\right) u_{2}(F(x))^{\lambda}
\end{aligned}
$$


Using Proposition 6, we conclude that

$$
v\left(F_{\tau}(x)\right) \geq u\left(F_{\tau}(x)\right)
$$

for all $x \in \hat{K}$. This implies

$$
\int_{M} v d \operatorname{vol}_{g(\tau)} \geq \int_{F_{\tau}(\hat{K})} v d \operatorname{vol}_{g(\tau)} \geq \int_{F_{\tau}(\hat{K})} u d \operatorname{vol}_{g(\tau)}=v\left(F_{\tau}(\hat{K})\right) .
$$

Moreover, we have

$$
v\left(F_{\tau}(\hat{K})\right)=v_{1}\left[F_{\tau}^{-1}\left(F_{\tau}(\hat{K})\right)\right] \geq v_{1}(\hat{K})=1
$$

by definition of $\nu$. Putting these facts together, the assertion follows.

\section{Relation to Perelman's reduced volume}

In this final section, we discuss how Theorem 2 is related to the monotonicity of Perelman's reduced volume. The strategy is to fix $\tau$ and $\tau_{2}$, and pass to the limit as $\tau_{1} \rightarrow 0$.

Let us fix a point $p \in M$ and real numbers $0<\tau<\tau_{2}<T$. We define a function $v$ by

$$
v=\tau^{-n / 2} \exp \left(-\frac{1}{2 \sqrt{\tau}} Q(p, 0 ; \cdot, \tau)\right) .
$$

For $\tau_{1}>0$ sufficiently small, we denote by $B\left(p, \sqrt{\tau_{1}}\right)$ the geodesic ball of radius $\sqrt{\tau_{1}}$ in the metric $g(0)$. We can find a positive constant $N$ such that $Q\left(p, 0 ; x, \tau_{1}\right) \leq N \sqrt{\tau_{1}}$ and $Q\left(x, \tau_{1} ; p, 2 \tau_{1}\right) \leq N \sqrt{\tau_{1}}$ for all points $x \in B\left(p, \sqrt{\tau_{1}}\right)$. Note that the constant $N$ is independent of $\tau_{1}$.

As above, we write

$$
\frac{1}{\sqrt{\tau}}=\frac{1-\lambda}{\sqrt{\tau_{1}}}+\frac{\lambda}{\sqrt{\tau_{2}}}
$$

where $0<\lambda<1$. We now specify the functions $u_{1}$ and $u_{2}$. We define

$$
\begin{aligned}
& u_{1}=\tau_{1}^{-n / 2} \exp \left(-\frac{N \sqrt{\tau_{1}}}{2(1-\lambda)}\left(\frac{1}{\sqrt{\tau}}+\frac{\lambda}{\sqrt{\tau_{2}}}\right)\right) \mathbb{1}_{B\left(p, \sqrt{\tau_{1}}\right)}, \\
& u_{2}=\tau_{2}^{-n / 2} \exp \left(-\frac{1}{2 \sqrt{\tau_{2}}} Q\left(p, 2 \tau_{1} ; \cdot, \tau_{2}\right)\right) .
\end{aligned}
$$

In the next step, we verify that $u_{1}, u_{2}, v$ satisfy the assumptions of Theorem 2

Proposition 8. We have

$$
\begin{aligned}
\left(\frac{\tau}{\tau_{1}^{1-\lambda} \tau_{2}^{\lambda}}\right)^{n / 2} v(\gamma(\tau)) \geq & \exp \left(-\frac{1-\lambda}{2 \sqrt{\tau_{1}}} Q\left(\gamma\left(\tau_{1}\right), \tau_{1} ; \gamma(\tau), \tau\right)\right) u_{1}\left(\gamma\left(\tau_{1}\right)\right)^{1-\lambda} \\
& \cdot \exp \left(\frac{\lambda}{2 \sqrt{\tau_{2}}} Q\left(\gamma(\tau), \tau ; \gamma\left(\tau_{2}\right), \tau_{2}\right)\right) u_{2}\left(\gamma\left(\tau_{2}\right)\right)^{\lambda}
\end{aligned}
$$

for every minimizing $\mathcal{L}$-geodesic $\gamma:\left[\tau_{1}, \tau_{2}\right] \rightarrow M$. 
Proof. If $\gamma(0) \notin B\left(p, \sqrt{\tau_{1}}\right)$, then $u_{1}(\gamma(0))=0$ and the assertion is trivial. Hence, it suffices to consider the case $\gamma(0) \in B\left(p, \sqrt{\tau_{1}}\right)$. In this case, we have

$$
\begin{aligned}
Q(p, 0 ; \gamma(\tau), \tau) & \leq Q\left(\gamma\left(\tau_{1}\right), \tau_{1} ; \gamma(\tau), \tau\right)+Q\left(p, 0 ; \gamma\left(\tau_{1}\right), \tau_{1}\right) \\
& \leq Q\left(\gamma\left(\tau_{1}\right), \tau_{1} ; \gamma(\tau), \tau\right)+N \sqrt{\tau_{1}}
\end{aligned}
$$

and

$$
\begin{aligned}
Q\left(p, 2 \tau_{1} ; \gamma\left(\tau_{2}\right), \tau_{2}\right) & \geq Q\left(\gamma\left(\tau_{1}\right), \tau_{1} ; \gamma\left(\tau_{2}\right), \tau_{2}\right)-Q\left(\gamma\left(\tau_{1}\right), \tau_{1} ; p, 2 \tau_{1}\right) \\
& \geq Q\left(\gamma\left(\tau_{1}\right), \tau_{1} ; \gamma\left(\tau_{2}\right), \tau_{2}\right)-N \sqrt{\tau_{1}} .
\end{aligned}
$$

This implies

$$
v(\gamma(\tau), \tau) \geq \tau^{-n / 2} \exp \left(-\frac{N \sqrt{\tau_{1}}}{2 \sqrt{\tau}}\right) \exp \left(-\frac{1}{2 \sqrt{\tau}} Q\left(\gamma\left(\tau_{1}\right), \tau_{1} ; \gamma(\tau), \tau\right)\right)
$$

and

$$
u_{2}\left(\gamma\left(\tau_{2}\right)\right) \leq \tau_{2}^{-n / 2} \exp \left(\frac{N \sqrt{\tau_{1}}}{2 \sqrt{\tau_{2}}}\right) \exp \left(-\frac{1}{2 \sqrt{\tau_{2}}} Q\left(\gamma\left(\tau_{1}\right), \tau_{1} ; \gamma\left(\tau_{2}\right), \tau_{2}\right)\right)
$$

Moreover, we have

$$
Q\left(\gamma\left(\tau_{1}\right), \tau_{1} ; \gamma\left(\tau_{2}\right), \tau_{2}\right)=Q\left(\gamma\left(\tau_{1}\right), \tau_{1} ; \gamma(\tau), \tau\right)+Q\left(\gamma(\tau), \tau ; \gamma\left(\tau_{2}\right), \tau_{2}\right)
$$

since $\gamma$ has minimal $\mathcal{L}$-length. Putting these facts together, we obtain

$$
\begin{aligned}
v(\gamma(\tau)) \geq & \left(\frac{\tau_{2}^{\lambda}}{\tau}\right)^{n / 2} \exp \left(-\frac{N \sqrt{\tau_{1}}}{2}\left(\frac{1}{\sqrt{\tau}}+\frac{\lambda}{\sqrt{\tau_{2}}}\right)\right) \\
& \cdot \exp \left(-\frac{1-\lambda}{2 \sqrt{\tau_{1}}} Q\left(\gamma\left(\tau_{1}\right), \tau_{1} ; \gamma(\tau), \tau\right)\right) \\
& \cdot \exp \left(\frac{\lambda}{2 \sqrt{\tau_{2}}} Q\left(\gamma(\tau), \tau ; \gamma\left(\tau_{2}\right), \tau_{2}\right)\right) u_{2}\left(\gamma\left(\tau_{2}\right)\right)^{\lambda}
\end{aligned}
$$

From this, the assertion follows.

Let $\tilde{V}(\tau)$ denote the reduced volume at time $\tau$. Using Theorem 2, we obtain

$$
\tilde{V}(\tau)=\int_{M} v d \operatorname{vol}_{g(\tau)} \geq\left(\int_{M} u_{1} d \operatorname{vol}_{g\left(\tau_{1}\right)}\right)^{1-\lambda}\left(\int_{M} u_{2} d \operatorname{vol}_{g\left(\tau_{2}\right)}\right)^{\lambda} .
$$

We now fix $\tau$ and $\tau_{2}$, and pass to the limit as $\tau_{1} \rightarrow 0$. Clearly,

$$
1-\lambda=\sqrt{\tau_{1}}\left(\frac{1}{\sqrt{\tau}}-\frac{1}{\sqrt{\tau_{2}}}\right)+O\left(\tau_{1}\right)
$$

This implies

$$
-\frac{N \sqrt{\tau_{1}}}{2(1-\lambda)}\left(\frac{1}{\sqrt{\tau}}+\frac{\lambda}{\sqrt{\tau_{2}}}\right) \rightarrow-\frac{N}{2}\left(\frac{1}{\sqrt{\tau}}-\frac{1}{\sqrt{\tau_{2}}}\right)^{-1}\left(\frac{1}{\sqrt{\tau}}+\frac{1}{\sqrt{\tau_{2}}}\right)
$$


as $\tau_{1} \rightarrow 0$. Hence, the integral $\int_{M} u_{1} d \operatorname{vol}_{g\left(\tau_{1}\right)}$ converges to a positive real number as $\tau_{1} \rightarrow 0$. Since $1-\lambda \rightarrow 0$, we conclude that

$$
\left(\int_{M} u_{1} d \operatorname{vol}_{g\left(\tau_{1}\right)}\right)^{1-\lambda} \rightarrow 1 \quad \text { as } \tau_{1} \rightarrow 0
$$

Moreover, we have

$$
\left(\int_{M} u_{2} d \operatorname{vol}_{g\left(\tau_{2}\right)}\right)^{\lambda} \rightarrow \tilde{V}\left(\tau_{2}\right) \quad \text { as } \tau_{1} \rightarrow 0 .
$$

Putting these facts together, we obtain

$$
\tilde{V}(\tau)=\int_{M} v d \operatorname{vol}_{g(\tau)} \geq \tilde{V}\left(\tau_{2}\right)
$$

Thus, Theorem 2 implies the monotonicity of the reduced volume.

Acknowledgments. The author was partially supported by the National Science Foundation under grant DMS-0905628.

\section{References}

[1] Brendle, S.: Ricci Flow and the Sphere Theorem. Grad. Stud. Math. 111, Amer. Math. Soc. (2010) Zbl pre05673930 MR 2583938

[2] Cordero-Erausquin, D., McCann, R., Schmuckenschläger, M.: A Riemannian interpolation inequality à la Borell, Brascamp, and Lieb. Invent. Math. 146, 219-257 (2001) Zbl 1026.58018 MR 1865396

[3] Cordero-Erausquin, D., McCann, R., Schmuckenschläger, M.: Prékopa-Leindler type inequalities on Riemannian manifolds, Jacobi fields, and optimal transport. Ann. Fac. Sci. Toulouse Math. 15, 613-635 (2006) Zbl 1125.58007 MR 2295207

[4] Hamilton, R.: Three-manifolds with positive Ricci curvature. J. Differential Geom. 17, 255306 (1982) Zbl 0504.53034 MR 0664497

[5] Leindler, L.: On a certain converse of Hölder's inequality. Acta Sci. Math. (Szeged) 33, $217-$ 233 (1972) Zbl 0245.26011 MR 2199372

[6] Lott, J.: Optimal transport and Perelman's reduced volume. Calc. Var. Partial Differential Equation 36, 49-84 (2009) Zbl 1171.53318 MR 2507614

[7] McCann, R., Topping, P.: Ricci flow, entropy and optimal transportation. Amer. J. Math. 132, 711-730 (2010) Zbl pre05724788 MR 2666905

[8] Perelman, G.: The entropy formula for the Ricci flow and its geometric applications. arXiv:0211159 Zbl 1130.53001

[9] Prékopa, A.: Logarithmic concave measures with applications to stochastic programming. Acta Sci. Math. (Szeged) 32, 301-315 (1971) Zbl 0235.90044 MR 0315079

[10] Topping, P.: Lectures on the Ricci Flow. London Math. Soc. Lecture Note Ser. 325, Cambridge Univ. Press, Cambridge (2006) Zbl 1105.58013 MR 2265040

[11] Topping, P.: $\mathcal{L}$-optimal transportation for Ricci flow. J. Reine Angew. Math. 636, 93-122 (2009) Zbl 1187.53072 MR 2572247

[12] Villani, C.: Optimal Transport, Old and New. Grundlehren Math. Wiss. 338, Springer, Berlin (2009) Zbl 1156.53003 MR 2459454 